\title{
Observation of the rare decay $B^{0} \rightarrow K_{S}^{0} K^{ \pm} \pi^{\mp}$
}

P. del Amo Sanchez, ${ }^{1}$ J. P. Lees, ${ }^{1}$ V. Poireau, ${ }^{1}$ E. Prencipe, ${ }^{1}$ V. Tisserand, ${ }^{1}$ J. Garra Tico, ${ }^{2}$ E. Grauges, ${ }^{2}$ M. Martinelli,,${ }^{3 a, 3 b}$ A. Palano, ${ }^{3 a, 3 b}$ M. Pappagallo, ${ }^{3 a, 3 b}$ G. Eigen, ${ }^{4}$ B. Stugu, ${ }^{4}$ L. Sun, ${ }^{4}$ M. Battaglia, ${ }^{5}$ D. N. Brown, ${ }^{5}$ B. Hooberman, ${ }^{5}$ L. T. Kerth, ${ }^{5}$ Yu. G. Kolomensky, ${ }^{5}$ G. Lynch, ${ }^{5}$ I. L. Osipenkov, ${ }^{5}$ T. Tanabe, ${ }^{5}$ C. M. Hawkes, ${ }^{6}$ N. Soni, ${ }^{6}$ A. T. Watson, ${ }^{6}$ H. Koch, ${ }^{7}$ T. Schroeder, ${ }^{7}$ D. J. Asgeirsson, ${ }^{8}$ C. Hearty ${ }^{8}$ T. S. Mattison, ${ }^{8}$ J. A. McKenna, ${ }^{8}$ A. Khan, ${ }^{9}$ A. Randle-Conde, ${ }^{9}$ V.E. Blinov,${ }^{10}$ A. R. Buzykaev, ${ }^{10}$ V. P. Druzhinin, ${ }^{10}$ V. B. Golubev, ${ }^{10}$ A. P. Onuchin, ${ }^{10}$ S. I. Serednyakov, ${ }^{10}$ Yu. I. Skovpen, ${ }^{10}$ E. P. Solodov,${ }^{10}$ K. Yu. Todyshev,${ }^{10}$ A. N. Yushkov, ${ }^{10}$ M. Bondioli, ${ }^{11}$ S. Curry, ${ }^{11}$ D. Kirkby,${ }^{11}$ A. J. Lankford, ${ }^{11}$ M. Mandelkern, ${ }^{11}$ E. C. Martin, ${ }^{11}$ D. P. Stoker, ${ }^{11}$ H. Atmacan, ${ }^{12}$ J. W. Gary, ${ }^{12}$ F. Liu, ${ }^{12}$ O. Long, ${ }^{12}$ G. M. Vitug, ${ }^{12}$ Z. Yasin, ${ }^{12}$ V. Sharma, ${ }^{13}$ C. Campagnari, ${ }^{14}$ T. M. Hong, ${ }^{14}$ D. Kovalskyi, ${ }^{14}$ J. D. Richman, ${ }^{14}$ A. M. Eisner, ${ }^{15}$ C. A. Heusch, ${ }^{15}$ J. Kroseberg, ${ }^{15}$ W. S. Lockman, ${ }^{15}$ A. J. Martinez, ${ }^{15}$ T. Schalk, ${ }^{15}$ B. A. Schumm, ${ }^{15}$ A. Seiden, ${ }^{15}$

L. O. Winstrom, ${ }^{15}$ C. H. Cheng, ${ }^{16}$ D. A. Doll, ${ }^{16}$ B. Echenard, ${ }^{16}$ D. G. Hitlin, ${ }^{16}$ P. Ongmongkolkul, ${ }^{16}$ F. C. Porter,${ }^{16}$ A. Y. Rakitin, ${ }^{16}$ R. Andreassen, ${ }^{17}$ M. S. Dubrovin, ${ }^{17}$ G. Mancinelli,,${ }^{17}$ B. T. Meadows, ${ }^{17}$ M. D. Sokoloff,${ }^{17}$ P. C. Bloom, ${ }^{18}$ W. T. Ford ${ }^{18}$ A. Gaz,${ }^{18}$ J. F. Hirschauer, ${ }^{18}$ M. Nagel,${ }^{18}$ U. Nauenberg, ${ }^{18}$ J. G. Smith, ${ }^{18}$ S. R. Wagner, ${ }^{18}$ R. Ayad, $,{ }^{19}, *$ W. H. Toki, ${ }^{19}$ A. Hauke, ${ }^{20}$ H. Jasper, ${ }^{20}$ T. M. Karbach, ${ }^{20}$ J. Merkel, ${ }^{20}$ A. Petzold,${ }^{20}$ B. Spaan, ${ }^{20}$ K. Wacker, ${ }^{20}$ M. J. Kobel, ${ }^{21}$ K. R. Schubert, ${ }^{21}$ R. Schwierz, ${ }^{21}$ D. Bernard, ${ }^{22}$ M. Verderi, ${ }^{22}$ P. J. Clark, ${ }^{23}$ S. Playfer, ${ }^{23}$ J. E. Watson, ${ }^{23}$ M. Andreotti, ${ }^{24 a, 24 b}$ D. Bettoni, ${ }^{24 a}$ C. Bozzi, ${ }^{24 a}$ R. Calabrese, ${ }^{24 a, 24 b}$ A. Cecchi, ${ }^{24 a, 24 b}$ G. Cibinetto, ${ }^{24 a, 24 b}$ E. Fioravanti, ${ }^{24 a, 24 b}$ P. Franchini, ${ }^{24 a, 24 b}$ E. Luppi,${ }^{24 a, 24 b}$ M. Munerato, ${ }^{24 a, 24 b}$ M. Negrini, ${ }^{24 a, 24 b}$ A. Petrella, ${ }^{24 a, 24 b}$ L. Piemontese, ${ }^{24 a}$ R. Baldini-Ferroli, ${ }^{25}$ A. Calcaterra, ${ }^{25}$ R. de Sangro, ${ }^{25}$ G. Finocchiaro, ${ }^{25}$ M. Nicolaci, ${ }^{25}$ S. Pacetti ${ }^{25}$ P. Patteri, ${ }^{25}$ I. M. Peruzzi, ${ }^{25, \dagger}$ M. Piccolo, ${ }^{25}$ M. Rama, ${ }^{25}$ A. Zallo, ${ }^{25}$ R. Contri, ${ }^{26 a, 26 b}$ E. Guido, ${ }^{26 a, 26 b}$ M. Lo Vetere ${ }^{26 a, 26 b}$ M. R. Monge, ${ }^{26 a, 26 b}$ S. Passaggio, ${ }^{26 a}$ C. Patrignani, ${ }^{26 a, 26 b}$ E. Robutti, ${ }^{26 a}$ S. Tosi, ${ }^{26 a, 26 b}$ B. Bhuyan, ${ }^{27}$ M. Morii, ${ }^{28}$ A. Adametz, ${ }^{29}$ J. Marks, ${ }^{29}$ S. Schenk, ${ }^{29}$ U. Uwer, ${ }^{29}$ F. U. Bernlochner, ${ }^{30}$ H. M. Lacker, ${ }^{30}$ T. Lueck, ${ }^{30}$ A. Volk, ${ }^{30}$ P. D. Dauncey, ${ }^{31}$ M. Tibbetts, ${ }^{31}$ P. K. Behera, ${ }^{32}$ U. Mallik, ${ }^{32}$ C. Chen, ${ }^{33}$ J. Cochran, ${ }^{33}$ H. B. Crawley,${ }^{33}$ L. Dong, ${ }^{33}$ W. T. Meyer, ${ }^{33}$ S. Prell, ${ }^{33}$ E. I. Rosenberg, ${ }^{33}$ A. E. Rubin, ${ }^{33}$ Y. Y. Gao, ${ }^{34}$ A. V. Gritsan, ${ }^{34}$ Z. J. Guo, ${ }^{34}$ N. Arnaud, ${ }^{35}$ M. Davier, ${ }^{35}$ D. Derkach,,${ }^{35}$ J. Firmino da Costa, ${ }^{35}$ G. Grosdidier, ${ }^{35}$ F. Le Diberder, ${ }^{35}$ A. M. Lutz, ${ }^{35}$ B. Malaescu, ${ }^{35}$ A. Perez, ${ }^{35}$ P. Roudeau, ${ }^{35}$ M. H. Schune, ${ }^{35}$ J. Serrano, ${ }^{35}$ V. Sordini, ${ }^{35,}$ A. Stocchi, ${ }^{35}$ L. Wang, ${ }^{35}$ G. Wormser, ${ }^{35}$ D. J. Lange, ${ }^{36}$ D. M. Wright, ${ }^{36}$ I. Bingham, ${ }^{37}$ J. P. Burke, ${ }^{37}$ C. A. Chavez, ${ }^{37}$ J. P. Coleman, ${ }^{37}$ J. R. Fry, ${ }^{37}$ E. Gabathuler, ${ }^{37}$ R. Gamet, ${ }^{37}$ D. E. Hutchcroft, ${ }^{37}$ D. J. Payne, ${ }^{37}$ C. Touramanis, ${ }^{37}$ A. J. Bevan, ${ }^{38}$ F. Di Lodovico, ${ }^{38}$ R. Sacco,${ }^{38}$ M. Sigamani, ${ }^{38}$ G. Cowan,${ }^{39}$ S. Paramesvaran, ${ }^{39}$ A. C. Wren, ${ }^{39}$ D. N. Brown, ${ }^{40}$ C. L. Davis, ${ }^{40}$ A. G. Denig, ${ }^{41}$ M. Fritsch,${ }^{41}$ W. Gradl,${ }^{41}$ A. Hafner, ${ }^{41}$ K. E. Alwyn, ${ }^{42}$ D. Bailey, ${ }^{42}$ R. J. Barlow, ${ }^{42}$ G. Jackson, ${ }^{42}$ G. D. Lafferty, ${ }^{42}$ T. J. West, ${ }^{42}$ J. Anderson, ${ }^{43}$ R. Cenci, ${ }^{43}$ A. Jawahery, ${ }^{43}$ D. A. Roberts, ${ }^{43}$ G. Simi,${ }^{43}$ J. M. Tuggle,${ }^{43}$ C. Dallapiccola ${ }^{44}$ E. Salvati ${ }^{44}$ R. Cowan, ${ }^{45}$ D. Dujmic, ${ }^{45}$ P. H. Fisher, ${ }^{45}$ G. Sciolla ${ }^{45}$ R. K. Yamamoto, ${ }^{45}$ M. Zhao, ${ }^{45}$ P. M. Patel, ${ }^{46}$ S. H. Robertson, ${ }^{46}$ M. Schram, ${ }^{46}$ P. Biassoni,${ }^{47 a, 47 b}$ A. Lazzaro, ${ }^{47 a, 47 b}$ V. Lombardo, ${ }^{47 a}$ F. Palombo, ${ }^{47 a, 47 b}$ S. Stracka, ${ }^{47 a, 47 b}$ L. Cremaldi, ${ }^{48}$ R. Godang, ${ }^{48,8}$ R. Kroeger, ${ }^{48}$ P. Sonnek, ${ }^{48}$ D. J. Summers ${ }^{48}$ H. W. Zhao ${ }^{48}$ X. Nguyen, ${ }^{49}$ M. Simard, ${ }^{49}$ P. Taras, ${ }^{49}$ G. De Nardo, ${ }^{50 a, 50 b}$ D. Monorchio, ${ }^{50 a, 50 b}$ G. Onorato, ${ }^{50 a, 50 b}$ C. Sciacca, ${ }^{50 a, 50 b}$ G. Raven, ${ }^{51}$ H. L. Snoek, ${ }^{51}$ C. P. Jessop, ${ }^{52}$ K. J. Knoepfel, ${ }^{52}$ J. M. LoSecco, ${ }^{52}$ W. F. Wang, ${ }^{52}$ L. A. Corwin, ${ }^{53}$ K. Honscheid, ${ }^{53}$ R. Kass, ${ }^{53}$ J. P. Morris, ${ }^{53}$ A. M. Rahimi,${ }^{53}$ N. L. Blount, ${ }^{54}$ J. Brau,${ }^{54}$ R. Frey,${ }^{54}$ O. Igonkina, ${ }^{54}$ J. A. Kolb,${ }^{54}$ R. Rahmat, ${ }^{54}$ N. B. Sinev, ${ }^{54}$ D. Strom,${ }^{54}$ J. Strube,${ }^{54}$ E. Torrence, ${ }^{54}$ G. Castelli, ${ }^{55 a, 55 b}$ E. Feltresi, ${ }^{55 a, 55 b}$ N. Gagliardi, ${ }^{55 a, 55 b}$ M. Margoni, ${ }^{55 a, 55 b}$ M. Morandin, ${ }^{55 a}$ M. Posocco, ${ }^{55 a}$ M. Rotondo, ${ }^{55 a}$ F. Simonetto, ${ }^{55,55 b}$ R. Stroili, ${ }^{55 a, 55 b}$ E. Ben-Haim, ${ }^{56}$ G. R. Bonneaud, ${ }^{56}$ H. Briand, ${ }^{56}$ J. Chauveau, ${ }^{56}$ O. Hamon, ${ }^{56}$ Ph. Leruste, ${ }^{56}$ G. Marchiori, ${ }^{56}$ J. Ocariz ${ }^{56}$ J. Prendki,${ }^{56}$ S. Sitt, ${ }^{56}$ M. Biasini, ${ }^{57 a, 57 b}$ E. Manoni, ${ }^{57 a, 57 b}$ C. Angelini ${ }^{58 a}, 58 b$ G. Batignani, ${ }^{58 \mathrm{a}, 58 \mathrm{~b}}$ S. Bettarini, ${ }^{58 \mathrm{a}, 58 \mathrm{~b}}$ G. Calderini, ${ }^{58 \mathrm{a}, 58 \mathrm{~b}, \|}$ M. Carpinelli, ${ }^{58 \mathrm{a}, 58 \mathrm{~b}, \mathrm{fl}}$ A. Cervelli, ${ }^{58 \mathrm{a}, 58 \mathrm{~b}}$ F. Forti, ${ }^{58 \mathrm{a}, 58 \mathrm{~b}}$ M. A. Giorgi, ${ }^{58 a, 58 b}$ A. Lusiani, ${ }^{58 a, 58 c}$ N. Neri, ${ }^{58 a, 58 b}$ E. Paoloni, ${ }^{58 a, 58 b}$ G. Rizzo, ${ }^{58 a, 58 b}$ J. J. Walsh, ${ }^{58 a}$ D. Lopes Pegna, ${ }^{59}$ C. Lu, ${ }^{59}$ J. Olsen, ${ }^{59}$ A. J. S. Smith, ${ }^{59}$ A. V. Telnov ${ }^{59}$ F. Anulli, ${ }^{60 a}$ E. Baracchini, ${ }^{60 a, 60 b}$ G. Cavoto, ${ }^{60 a}$ R. Faccini, ${ }^{60 a, 60 b}$ F. Ferrarotto, ${ }^{60 \mathrm{a}}$ F. Ferroni, ${ }^{60 \mathrm{a}, 60 \mathrm{~b}}$ M. Gaspero, ${ }^{60 \mathrm{a}, 60 \mathrm{~b}}$ L. Li Gioi ${ }^{60 \mathrm{a}}$ M. A. Mazzoni, ${ }^{60 \mathrm{a}}$ G. Piredda ${ }^{60 \mathrm{a}}$ F. Renga, ${ }^{60 \mathrm{a}, 60 \mathrm{~b}}$ M. Ebert, ${ }^{61}$ T. Hartmann, ${ }^{61}$ T. Leddig, ${ }^{61}$ H. Schröder, ${ }^{61}$ R. Waldi, ${ }^{61}$ T. Adye,${ }^{62}$ B. Franek, ${ }^{62}$ E. O. Olaiya, ${ }^{62}$ F. F. Wilson, ${ }^{62}$ S. Emery, ${ }^{63}$ G. Hamel de Monchenault, ${ }^{63}$ G. Vasseur, ${ }^{63}$ Ch. Yèche, ${ }^{63}$ M. Zito, ${ }^{63}$ M. T. Allen, ${ }^{64}$ D. Aston, ${ }^{64}$ D. J. Bard, ${ }^{64}$ R. Bartoldus, ${ }^{64}$ J. F. Benitez, ${ }^{64}$ C. Cartaro,${ }^{64}$ M. R. Convery ${ }^{64}$ J. Dorfan, ${ }^{64}$ G. P. Dubois-Felsmann,${ }^{64}$ W. Dunwoodie, ${ }^{64}$

R. C. Field ${ }^{64}$ M. Franco Sevilla, ${ }^{64}$ B. G. Fulsom, ${ }^{64}$ A. M. Gabareen, ${ }^{64}$ M. T. Graham, ${ }^{64}$ P. Grenier, ${ }^{64}$ C. Hast, ${ }^{64}$ W. R. Innes, ${ }^{64}$ M. H. Kelsey, ${ }^{64}$ H. Kim, ${ }^{64}$ P. Kim,${ }^{64}$ M. L. Kocian, ${ }^{64}$ D. W. G. S. Leith, ${ }^{64}$ S. Li, ${ }^{64}$ B. Lindquist, ${ }^{64}$ S. Luitz, ${ }^{64}$ V. Luth ${ }^{64}$ H. L. Lynch, ${ }^{64}$ D. B. MacFarlane ${ }^{64}$ H. Marsiske, ${ }^{64}$ D. R. Muller,${ }^{64}$ H. Neal, ${ }^{64}$ S. Nelson, ${ }^{64}$ C. P. O' Grady, ${ }^{64}$ I. Ofte, ${ }^{64}$ M. Perl, ${ }^{64}$ B. N. Ratcliff, ${ }^{64}$ A. Roodman, ${ }^{64}$ A. A. Salnikov, ${ }^{64}$ R. H. Schindler, ${ }^{64}$ J. Schwiening, ${ }^{64}$ A. Snyder, ${ }^{64}$ 
D. Su, ${ }^{64}$ M. K. Sullivan, ${ }^{64}$ K. Suzuki, ${ }^{64}$ J. M. Thompson, ${ }^{64}$ J. Va'vra, ${ }^{64}$ A. P. Wagner, ${ }^{64}$ M. Weaver, ${ }^{64}$ C. A. West,${ }^{64}$ W. J. Wisniewski ${ }^{64}$ M. Wittgen, ${ }^{64}$ D. H. Wright,${ }^{64}$ H. W. Wulsin, ${ }^{64}$ A. K. Yarritu, ${ }^{64}$ V. Santoro, ${ }^{64}$ C. C. Young, ${ }^{64}$ V. Ziegler ${ }^{64}$ X. R. Chen ${ }^{65}$ W. Park,,${ }^{65}$ M. V. Purohit,,${ }^{65}$ R. M. White, ${ }^{65}$ J. R. Wilson, ${ }^{65}$ S. J. Sekula, ${ }^{66}$ M. Bellis, ${ }^{67}$ P. R. Burchat, ${ }^{67}$ A. J. Edwards, ${ }^{67}$ T. S. Miyashita, ${ }^{67}$ S. Ahmed ${ }^{68}$ M. S. Alam, ${ }^{68}$ J. A. Ernst, ${ }^{68}$ B. Pan, ${ }^{68}$ M. A. Saeed, ${ }^{68}$ S. B. Zain, ${ }^{68}$ N. Guttman, ${ }^{69}$ A. Soffer,${ }^{69}$ P. Lund, ${ }^{70}$ S. M. Spanier, ${ }^{70}$ R. Eckmann, ${ }^{71}$ J. L. Ritchie, ${ }^{71}$ A. M. Ruland, ${ }^{71}$ C. J. Schilling, ${ }^{71}$ R. F. Schwitters, ${ }^{71}$ B. C. Wray, ${ }^{71}$ J. M. Izen, ${ }^{72}$ X. C. Lou, ${ }^{72}$ F. Bianchi, ${ }^{73 a, 73 b}$ D. Gamba, ${ }^{73 a, 73 b}$ M. Pelliccioni, ${ }^{73 a, 73 b}$ M. Bomben, ${ }^{74 a, 74 b}$ G. Della Ricca, ${ }^{74 a, 74 b}$ L. Lanceri, ${ }^{74 a, 74 b}$ L. Vitale,${ }^{74 a, 74 b}$ V. Azzolini, ${ }^{75}$ N. Lopez-March, ${ }^{75}$ F. Martinez-Vidal, ${ }^{75}$ D. A. Milanes, ${ }^{75}$ A. Oyanguren, ${ }^{75}$ J. Albert, ${ }^{76}$ Sw. Banerjee ${ }^{76}$ H. H. F. Choi, ${ }^{76}$ K. Hamano, ${ }^{76}$ G. J. King, ${ }^{76}$ R. Kowalewski, ${ }^{76}$ M. J. Lewczuk, ${ }^{76}$ I. M. Nugent, ${ }^{76}$ J. M. Roney, ${ }^{76}$ R. J. Sobie, ${ }^{76}$ T. J. Gershon, ${ }^{77}$ P. F. Harrison, ${ }^{77}$ J. Ilic, ${ }^{77, * *}$ T. E. Latham, ${ }^{77}$ G. B. Mohanty, ${ }^{77, \dagger \dagger}$ E. M. T. Puccio, ${ }^{77}$ H. R. Band, ${ }^{78}$ X. Chen, ${ }^{78}$ S. Dasu, ${ }^{78}$ K. T. Flood, ${ }^{78}$ Y. Pan, ${ }^{78}$ R. Prepost ${ }^{78}$ C. O. Vuosalo, ${ }^{78}$ and S. L. $\mathrm{Wu}^{78}$

(BABAR Collaboration)

\author{
${ }^{1}$ Laboratoire d'Annecy-le-Vieux de Physique des Particules (LAPP), Université de Savoie, \\ CNRS/IN2P3, F-74941 Annecy-Le-Vieux, France \\ ${ }^{2}$ Universitat de Barcelona, Facultat de Fisica, Departament ECM, E-08028 Barcelona, Spain \\ ${ }^{3}$ INFN Sezione di Bari, I-70126 Bari, Italy \\ ${ }^{3 \mathrm{~b}}$ Dipartimento di Fisica, Università di Bari, I-70126 Bari, Italy \\ ${ }^{4}$ University of Bergen, Institute of Physics, N-5007 Bergen, Norway \\ ${ }^{5}$ Lawrence Berkeley National Laboratory and University of California, Berkeley, California 94720, USA \\ ${ }^{6}$ University of Birmingham, Birmingham, B15 2TT, United Kingdom \\ ${ }^{7}$ Ruhr Universität Bochum, Institut für Experimentalphysik 1, D-44780 Bochum, Germany \\ ${ }^{8}$ University of British Columbia, Vancouver, British Columbia, Canada V6T 1Z1 \\ ${ }^{9}$ Brunel University, Uxbridge, Middlesex UB8 3PH, United Kingdom \\ ${ }^{10}$ Budker Institute of Nuclear Physics, Novosibirsk 630090, Russia \\ ${ }^{11}$ University of California at Irvine, Irvine, California 92697, USA \\ ${ }^{12}$ University of California at Riverside, Riverside, California 92521, USA \\ ${ }^{13}$ University of California at San Diego, La Jolla, California 92093, USA \\ ${ }^{14}$ University of California at Santa Barbara, Santa Barbara, California 93106, USA \\ ${ }^{15}$ University of California at Santa Cruz, Institute for Particle Physics, Santa Cruz, California 95064, USA \\ ${ }^{16}$ California Institute of Technology, Pasadena, California 91125, USA \\ ${ }^{17}$ University of Cincinnati, Cincinnati, Ohio 45221, USA \\ ${ }^{18}$ University of Colorado, Boulder, Colorado 80309, USA \\ ${ }^{19}$ Colorado State University, Fort Collins, Colorado 80523, USA \\ ${ }^{20}$ Technische Universität Dortmund, Fakultät Physik, D-44221 Dortmund, Germany \\ ${ }^{21}$ Technische Universität Dresden, Institut für Kern- und Teilchenphysik, D-01062 Dresden, Germany \\ ${ }^{22}$ Laboratoire Leprince-Ringuet, CNRS/IN2P3, Ecole Polytechnique, F-91128 Palaiseau, France \\ ${ }^{23}$ University of Edinburgh, Edinburgh EH9 3JZ, United Kingdom \\ ${ }^{24 a}$ INFN Sezione di Ferrara, I-44100 Ferrara, Italy \\ ${ }^{24 \mathrm{~b}}$ Dipartimento di Fisica, Università di Ferrara, I-44100 Ferrara, Italy \\ ${ }^{25}$ INFN Laboratori Nazionali di Frascati, I-00044 Frascati, Italy \\ ${ }^{26 a}$ INFN Sezione di Genova, I-16146 Genova, Italy \\ ${ }^{26 \mathrm{~b}}$ Dipartimento di Fisica, Università di Genova, I-16146 Genova, Italy \\ ${ }^{27}$ Indian Institute of Technology Guwahati, Guwahati, Assam, 781 039, India \\ ${ }^{28}$ Harvard University, Cambridge, Massachusetts 02138, USA \\ ${ }^{29}$ Universität Heidelberg, Physikalisches Institut, Philosophenweg 12, D-69120 Heidelberg, Germany \\ ${ }^{30}$ Humboldt-Universität zu Berlin, Institut für Physik, Newtonstr. 15, D-12489 Berlin, Germany \\ ${ }^{31}$ Imperial College London, London, SW7 2AZ, United Kingdom \\ ${ }^{32}$ University of Iowa, Iowa City, Iowa 52242, USA \\ ${ }^{33}$ Iowa State University, Ames, Iowa 50011-3160, USA \\ ${ }^{34}$ Johns Hopkins University, Baltimore, Maryland 21218, USA \\ ${ }^{35}$ Laboratoire de l'Accélérateur Linéaire, IN2P3/CNRS et Université Paris-Sud 11, Centre Scientifique d'Orsay, \\ B. P. 34, F-91898 Orsay Cedex, France \\ ${ }^{36}$ Lawrence Livermore National Laboratory, Livermore, California 94550, USA \\ ${ }^{37}$ University of Liverpool, Liverpool L69 7ZE, United Kingdom \\ ${ }^{38}$ Queen Mary, University of London, London, E1 4NS, United Kingdom \\ ${ }^{39}$ University of London, Royal Holloway and Bedford New College, Egham, Surrey TW20 OEX, United Kingdom
}




\author{
${ }^{40}$ University of Louisville, Louisville, Kentucky 40292, USA \\ ${ }^{41}$ Johannes Gutenberg-Universität Mainz, Institut für Kernphysik, D-55099 Mainz, Germany \\ ${ }^{42}$ University of Manchester, Manchester M13 9PL, United Kingdom \\ ${ }^{43}$ University of Maryland, College Park, Maryland 20742, USA \\ ${ }^{44}$ University of Massachusetts, Amherst, Massachusetts 01003, USA \\ ${ }^{45}$ Massachusetts Institute of Technology, Laboratory for Nuclear Science, Cambridge, Massachusetts 02139, USA \\ ${ }^{46}$ Mc Gill University, Montréal, Québec, Canada H3A $2 T 8$ \\ ${ }^{47 \mathrm{a}}$ INFN Sezione di Milano, I-20133 Milano, Italy \\ ${ }^{47 b}$ Dipartimento di Fisica, Università di Milano, I-20133 Milano, Italy \\ ${ }^{48}$ University of Mississippi, University, Mississippi 38677, USA \\ ${ }^{49}$ Université de Montréal, Physique des Particules, Montréal, Québec, Canada H3C 3J7 \\ ${ }^{50 a}$ INFN Sezione di Napoli, I-80126 Napoli, Italy \\ ${ }^{50 \mathrm{~b}}$ Dipartimento di Scienze Fisiche, Università di Napoli Federico II, I-80126 Napoli, Italy \\ ${ }^{51}$ NIKHEF, National Institute for Nuclear Physics and High Energy Physics, NL-1009 DB Amsterdam, The Netherlands \\ ${ }^{52}$ University of Notre Dame, Notre Dame, Indiana 46556, USA \\ ${ }^{53}$ Ohio State University, Columbus, Ohio 43210, USA \\ ${ }^{54}$ University of Oregon, Eugene, Oregon 97403, USA \\ ${ }^{55}$ INFN Sezione di Padova, I-35131 Padova, Italy \\ ${ }^{55 b}$ Dipartimento di Fisica, Università di Padova, I-35131 Padova, Italy \\ ${ }^{56}$ Laboratoire de Physique Nucléaire et de Hautes Energies, IN2P3/CNRS, Université Pierre et Marie Curie-Paris6, \\ Université Denis Diderot-Paris7, F-75252 Paris, France \\ ${ }^{57 a}$ INFN Sezione di Perugia, I-06100 Perugia, Italy \\ ${ }^{57 b}$ Dipartimento di Fisica, Università di Perugia, I-06100 Perugia, Italy \\ ${ }^{58 a}$ INFN Sezione di Pisa, I-56127 Pisa, Italy \\ ${ }^{58 \mathrm{~b}}$ Dipartimento di Fisica, Università di Pisa, I-56127 Pisa, Italy \\ ${ }^{58 \mathrm{c}}$ Scuola Normale Superiore di Pisa, I-56127 Pisa, Italy \\ ${ }^{59}$ Princeton University, Princeton, New Jersey 08544, USA \\ ${ }^{60 a}$ INFN Sezione di Roma, I-00185 Roma, Italy \\ ${ }^{60 \mathrm{~b}}$ Dipartimento di Fisica, Università di Roma La Sapienza, I-00185 Roma, Italy \\ ${ }^{61}$ Universität Rostock, D-18051 Rostock, Germany \\ ${ }^{62}$ Rutherford Appleton Laboratory, Chilton, Didcot, Oxon, OX11 OQX, United Kingdom \\ ${ }^{63}$ CEA, Irfu, SPP, Centre de Saclay, F-91191 Gif-sur-Yvette, France \\ ${ }^{64}$ SLAC National Accelerator Laboratory, Stanford, California 94309 USA \\ ${ }^{65}$ University of South Carolina, Columbia, South Carolina 29208, USA \\ ${ }^{66}$ Southern Methodist University, Dallas, Texas 75275, USA \\ ${ }^{67}$ Stanford University, Stanford, California 94305-4060, USA \\ ${ }^{68}$ State University of New York, Albany, New York 12222, USA \\ ${ }^{69}$ Tel Aviv University, School of Physics and Astronomy, Tel Aviv, 69978, Israel \\ ${ }^{70}$ University of Tennessee, Knoxville, Tennessee 37996, USA \\ ${ }^{71}$ University of Texas at Austin, Austin, Texas 78712, USA \\ ${ }^{72}$ University of Texas at Dallas, Richardson, Texas 75083, USA \\ ${ }^{73 a}$ INFN Sezione di Torino, I-10125 Torino, Italy \\ ${ }^{73 b}$ Dipartimento di Fisica Sperimentale, Università di Torino, I-10125 Torino, Italy \\ ${ }^{74 a}$ INFN Sezione di Trieste, I-34127 Trieste, Italy \\ ${ }^{74 \mathrm{~b}}$ Dipartimento di Fisica, Università di Trieste, I-34127 Trieste, Italy \\ ${ }^{75}$ IFIC, Universitat de Valencia-CSIC, E-46071 Valencia, Spain \\ ${ }^{76}$ University of Victoria, Victoria, British Columbia, Canada V8W $3 P 6$ \\ ${ }^{77}$ Department of Physics, University of Warwick, Coventry CV4 7AL, United Kingdom \\ ${ }^{78}$ University of Wisconsin, Madison, Wisconsin 53706, USA
}

(Received 2 March 2010; published 5 August 2010)

\footnotetext{
*Now at Temple University, Philadelphia, Pennsylvania 19122, USA.

${ }^{\dagger}$ Also with Università di Perugia, Dipartimento di Fisica, Perugia, Italy.

*Also with Università di Roma La Sapienza, I-00185 Roma, Italy.

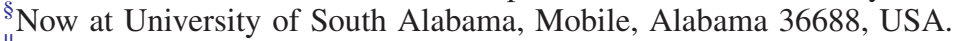
Université Denis Diderot-Paris7, F-75252 Paris, France.

II Also with Università di Sassari, Sassari, Italy.

**Now at Rutherford Appleton Laboratory, Chilton, Didcot, Oxon, OX11 0QX, United Kingdom.

${ }^{\dagger}$ Now at Tata Institute of Fundamental Research, Colaba, Mumbai 400 005, India.
}

"Also with Laboratoire de Physique Nucléaire et de Hautes Energies, IN2P3/CNRS, Université Pierre et Marie Curie-Paris6, 
We report an analysis of charmless hadronic decays of neutral $B$ mesons to the final state $K_{S}^{0} K^{ \pm} \pi^{\mp}$, using a data sample of $(465 \pm 5) \times 10^{6} B \bar{B}$ events collected with the $B A B A R$ detector at the $Y(4 S)$ resonance. We observe an excess of signal events with a significance of 5.2 standard deviations including systematic uncertainties and measure the branching fraction to be $\mathcal{B}\left(B^{0} \rightarrow K_{S}^{0} K^{ \pm} \pi^{\mp}\right)=(3.2 \pm 0.5 \pm$ $0.3) \times 10^{-6}$, where the uncertainties are statistical and systematic, respectively.

Charmless decays of $B$ mesons to hadronic final states containing an even number of kaons are suppressed in the standard model (SM). Decays of this type mainly proceed via the $b \rightarrow d$ "penguin" transition, involving a virtual loop, and hence are sensitive to potential new physics contributions since the presence of new particles in the loops can produce deviations from SM expectations. In recent years, there has been a surge of new results on these decays: $B^{0} \rightarrow K_{S}^{0} K_{S}^{0}$ and $B^{+} \rightarrow K_{S}^{0} K^{+}$have been observed $[1,2]$, and there is evidence for the related vector-vector final states [3-5]. Only upper limits on the corresponding pseudoscalar-vector final states exist: $\mathcal{B}\left(B^{0} \rightarrow K^{0} \bar{K}^{* 0}\right)+$ $\mathcal{B}\left(B^{0} \rightarrow \bar{K}^{0} K^{* 0}\right)<1.9 \times 10^{-6} \quad[6] \quad$ and $\quad \mathcal{B}\left(B^{+} \rightarrow\right.$ $\left.K^{+} \bar{K}^{* 0}\right)<1.1 \times 10^{-6}$ [7], both at $90 \%$ confidence level (unless explicitly stated otherwise we use the symbol $K^{*}$ to denote the $K^{*}(892)$ resonance and the inclusion of charge conjugate modes is implied). Note that decays with additional suppression in the SM, such as $B^{0} \rightarrow K^{(*)+} K^{(*)-}$, which are expected to proceed via annihilation amplitudes, have not been observed [2,3,8-13].

Since the vector resonances involved have nonnegligible widths, the pseudoscalar-vector decays are best studied using Dalitz plots of the three-body $K K \pi$ final states. In the three-body channels, contributions from suppressed $b \rightarrow u$ tree amplitudes are expected to be important, in addition to the $b \rightarrow d$ penguin amplitudes. Recent investigations of three-body channels suggest that additional resonances are present. Most notably, the $B^{+} \rightarrow$ $K^{+} K^{-} \pi^{+}$channel exhibits an unexpected peak near $1.5 \mathrm{GeV} / c^{2}$ in the $K^{+} K^{-}$invariant-mass spectrum, which accounts for approximately half of the total event rate [14]. We call this peak, with unknown spin and isospin quantum numbers, the $f_{\mathrm{X}}(1500)$. The lack of a $f_{\mathrm{X}}(1500)$ signal in $B^{+} \rightarrow K_{S}^{0} K_{S}^{0} \pi^{+}$decays implies that the $f_{X}(1500)$ does not have even spin if isospin is conserved in the decay [15]. A search for an isospin partner to the $f_{\mathrm{X}}(1500)$ that decays to $\bar{K}^{0} K^{+}$and which could be produced recoiling against a pion in $B$ decay could help to clarify the nature of this resonance.

In this paper, we present the results of a search for the three-body decay $B^{0} \rightarrow K_{S}^{0} K^{ \pm} \pi^{\mp}$, including intermediate two-body modes that decay to this final state but do not contain charm quarks. No decays to this final state have been observed as yet. The best available upper limit on the inclusive branching fraction is $\mathcal{B}\left(B^{0} \rightarrow K^{0} K^{ \pm} \pi^{\mp}\right)<18 \times$ $10^{-6}$ [16]. There appears to be no explicit prediction for the inclusive branching fraction of $B^{0} \rightarrow K_{S}^{0} K^{ \pm} \pi^{\mp}$. Some theoretical predictions exist, however, for the relevant resonant modes. Expected branching fractions for $B^{0} \rightarrow$ $\left(\bar{K}^{* 0} K^{0}+K^{* 0} \bar{K}^{0}\right)$ and $B^{0} \rightarrow K^{* \pm} K^{\mp}$ are in the range $(0.2-2.0) \times 10^{-6}$ and $(0.2-1.0) \times 10^{-7}$, respectively [1724]. Extensions to the SM can yield significantly larger branching fractions. For instance, in supersymmetric models with $R$-parity violation, the branching fraction for $B^{0} \rightarrow$ $\left(\bar{K}^{* 0} K^{0}+K^{* 0} \bar{K}^{0}\right)$ could be as large as $10^{-5}$ [25].

The data used in the analysis, collected with the BABAR detector [26] at the PEP-II asymmetric energy $e^{+} e^{-}$collider at SLAC, consist of an integrated luminosity of $424 \mathrm{fb}^{-1}$ recorded at the $Y(4 S)$ resonance ("on-peak") and $44 \mathrm{fb}^{-1}$ collected $40 \mathrm{MeV}$ below the resonance ("off-peak"). The on-peak data sample contains (465 \pm 5) $\times 10^{6} B \bar{B}$ events.

We reconstruct $B^{0} \rightarrow K_{S}^{0} K^{ \pm} \pi^{\mp}$ decay candidates by combining a $K_{S}^{0}$ candidate with one charged kaon and one oppositely charged pion candidate. The $K^{ \pm}$and $\pi^{ \pm}$ candidates are required to have a minimum transverse momentum of $50 \mathrm{MeV} / c$ and to be consistent with having originated from the interaction region. Identification of charged kaons and pions is accomplished with energyloss information from the tracking subdetectors, and the Cherenkov angle and number of photons measured by a ring-imaging Cherenkov detector. We distinguish kaons from pions by applying criteria to the product of the likelihood ratios determined from these individual measurements. The efficiency for kaon selection is approximately $80 \%$ including geometrical acceptance, while the probability of misidentification of pions as kaons is below $5 \%$ up to a laboratory momentum of $4 \mathrm{GeV} / c$. A $K_{S}^{0} \rightarrow \pi^{+} \pi^{-}$ candidate is formed from a pair of oppositely charged tracks (with the pion mass hypothesis assumed) having an invariant mass that lies within $15 \mathrm{MeV} / c^{2}$ of the nominal $K_{S}^{0}$ mass [27], corresponding to 5 times the $K_{S}^{0}$ mass resolution. We require the ratio of the measured $K_{S}^{0}$ decay length and its uncertainty to be greater than 20 , the cosine of the angle between the line connecting the $B$ and $K_{S}^{0}$ decay vertices and the $K_{S}^{0}$ momentum vector to be greater than 0.999 , and the $K_{S}^{0}$ vertex fit probability to be greater than $10^{-6}$.

To suppress the dominant background contribution, which arises from continuum $e^{+} e^{-} \rightarrow q \bar{q}(q=u, d, s$, c) events, we employ a Fisher discriminant that combines four variables. These are the ratio of the second to the zeroth order momentum-weighted angular moment [28], the absolute value of the cosine of the angle between the $B$ 
direction and the beam axis, the magnitude of the cosine of the angle between the $B$ thrust axis and the beam axis, and the proper time difference between the decays of the two $B$ mesons divided by its statistical uncertainty. The first three quantities are calculated in the center-of-mass (CM) frame.

In addition to the Fisher output $(\mathcal{F})$, we distinguish signal from background events using two kinematic variables: the difference $\Delta E$ between the CM energy of the $B$ candidate and $\sqrt{s} / 2$, and the beam-energy-substituted mass $m_{\mathrm{ES}}=\sqrt{s / 4-\mathbf{p}_{B}^{2}}$, where $\sqrt{s}$ is the total CM energy and $\mathbf{p}_{B}$ is the momentum of the candidate $B$ meson in the $\mathrm{CM}$ frame. The signal $m_{\mathrm{ES}}$ distribution peaks near the $B$ mass with a resolution of about $2.6 \mathrm{MeV} / c^{2}$, while its $\Delta E$ distribution peaks at zero with a resolution of approximately $20 \mathrm{MeV}$. We select signal candidates that satisfy $5.272<m_{\mathrm{ES}}<5.286 \mathrm{GeV} / c^{2},|\Delta E|<0.075 \mathrm{GeV}$, and $\mathcal{F}>-0.145$. The requirement on $\mathcal{F}$ removes approximately $70 \%$ of continuum background while retaining $90 \%$ of signal events.

Another source of background arises from $B$ decays, mostly involving intermediate charm or charmonium mesons, or charmless final states that are misreconstructed. We exclude $B$ candidates that have two-body mass combinations in any of the following invariant-mass ranges: $1.82<m\left(K_{S}^{0} K^{ \pm}\right)<2.04, \quad 1.81<m\left(K_{S}^{0} \pi^{\mp}\right)<1.91$, $1.83<m\left(K^{ \pm} \pi^{\mp}\right)<1.90,3.06<m\left(K^{ \pm} \pi^{\mp}\right)<3.17$, and $3.66<m\left(K^{ \pm} \pi^{\mp}\right)<3.73$ (all in units of $\mathrm{GeV} / c^{2}$ ). These ranges reject decays from $D^{+}$and $D_{s}^{+}, D^{+}, D^{0}, J / \psi$, and $\psi(2 S)$ mesons, respectively. Charmonium contributions result mainly from the leptonic decays of $J / \psi$ and $\psi(2 S)$, where one lepton is misidentified as a charged pion and the other as a kaon.

The efficiency for signal events to pass all the selection criteria is determined as a function of position in the Dalitz plot. Using a Monte Carlo (MC) simulation in which events uniformly populate the phase-space, we obtain an average efficiency of 20\%, though values as high as double (as low as half) that value are found near the center (corners) of the Dalitz plot.

An average of $1.1 \mathrm{~B}$ candidates is found per selected event. In events with multiple candidates we choose the one with the highest $B$ vertex fit probability. We verify that this procedure does not bias our fit variables. In some signal events, the $B$ candidate is misreconstructed due to one track being replaced with a track from the rest of the event. The fraction of such events is below $2 \%$ in the phase-space $\mathrm{MC}$, but is closer to 5\% in MC samples where the events populate the $K^{*}$ bands. Misreconstructed signal events are considered as a part of the signal component in the fit described below. We assign a systematic error to account for the uncertainty in the rate of these events, which is related to the unknown Dalitz-plot distribution of the $B^{0} \rightarrow K_{S}^{0} K^{ \pm} \pi^{\mp}$ decay.

We study residual background contributions from $B \bar{B}$ events that survive the invariant-mass exclusion require- ments described earlier, using MC simulations. It is found that these events can be combined into four categories based on their shapes in $m_{\mathrm{ES}}$ and $\Delta E$. The first category $\left(B \bar{B}_{1}\right)$ comprises $B^{0} \rightarrow \eta^{\prime} K_{S}^{0}, \eta^{\prime} \rightarrow \rho^{0} \gamma$ and misreconstructed $B^{0} \rightarrow D^{-} \pi^{+}, D^{-} \rightarrow K_{S}^{0} K^{-}$decays and has a broad peak in $m_{\mathrm{ES}}$ and a nonpeaking $\Delta E$ shape. The second and third categories $\left(B \bar{B}_{2}\right.$ and $\left.B \bar{B}_{3}\right)$ represent the charmless decays $B^{0} \rightarrow K_{S}^{0} K^{+} K^{-}$and $B^{0} \rightarrow K_{S}^{0} \pi^{+} \pi^{-}$, where a kaon or a pion is misidentified leading to a $\Delta E$ distribution that peaks with negative or positive mean, respectively. The MC simulations of these decays are based on our recent studies of their Dalitz plot distributions $[29,30]$. The fourth category $\left(B \bar{B}_{4}\right)$ contains the remainder of the $B \bar{B}$ background and is mainly combinatorial in nature. Based on the MC-derived efficiencies, total number of $B \bar{B}$ events, and known branching fractions [27,31], we expect 25, 173, 215 , and 668 events from the four $B \bar{B}$ background categories, respectively.

To obtain the $B^{0} \rightarrow K_{S}^{0} K^{ \pm} \pi^{\mp}$ signal yield, we perform an unbinned extended maximum likelihood fit to the candidate events using three input variables: $m_{\mathrm{ES}}, \Delta E$, and $\mathcal{F}$. These variables are found to be largely uncorrelated-the maximum correlation is between the signal $m_{\mathrm{ES}}$ and $\Delta E$ distributions and is about $13 \%$. For each component $j$ (signal, $q \bar{q}$ background, and the four $B \bar{B}$ background categories), we define a probability density function (PDF)

$$
\mathcal{P}_{j}^{i} \equiv \mathcal{P}_{j}\left(m_{\mathrm{ES}}{ }^{i}\right) \mathcal{P}_{j}\left(\Delta E^{i}\right) \mathcal{P}_{j}\left(\mathcal{F}^{i}\right),
$$

where $i$ denotes the event index. The extended likelihood function is given as

$$
\mathcal{L}=\prod_{k} e^{-n_{k}} \prod_{i}\left[\sum_{j} n_{j} \mathcal{P}_{j}^{i}\right],
$$

where $n_{j(k)}$ is the yield of the event category $j(k)$.

For the signal component, the $m_{\mathrm{ES}}$ and $\Delta E$ distributions are each parametrized by the sum of two Gaussian functions, while the $\mathcal{F}$ distribution is described by a bifurcated Gaussian function with a small admixture from the sum of two Gaussians. We fix the shape parameters to the values obtained from the $B^{0} \rightarrow K_{S}^{0} K^{ \pm} \pi^{+}$phase-space MC sample, after adjusting them to account for possible differences between data and MC simulations determined with a control sample of $B^{0} \rightarrow D^{-} \pi^{+}, D^{-} \rightarrow K_{S}^{0} \pi^{-}$decays. For the continuum background, we use an ARGUS function [32] to parametrize the $m_{\mathrm{ES}}$ shape and a linear function for $\Delta E$. The continuum Fisher shape is modeled with a function that is composed of a Gaussian tail with relative fraction 99.6\% (large component) and a small Gaussian with different mean and width values. This shape provides a good description of the off-peak Fisher distribution, as well as of the corresponding MC distribution. One-dimensional histograms are used as nonparametric PDFs to represent all three fit variables for the four $B \bar{B}$ background components.

The free parameters of our fit are the yields of signal, $B \bar{B}_{2}, B \bar{B}_{3}$, and continuum background together with the 
slope of the continuum $\Delta E$ PDF and the mean and width of the large Gaussian component of the continuum $\mathcal{F}$ PDF. The ARGUS $\xi$ parameter and parameters of the small Gaussian component of the continuum Fisher function are fixed to values determined from candidates selected in the off-peak data sample with a looser requirement on $m_{\mathrm{ES}}$. The yields of $B \bar{B}_{1}$ and $B \bar{B}_{4}$, and all shape parameters of the four $B \bar{B}$ background categories are fixed to the values determined from $\mathrm{MC}$ simulations.

We cross check our analysis procedure by removing the requirements that reject backgrounds from $B$ decays involving charm mesons, instead selecting regions of the Dalitz plot dominated by intermediate charm mesons. We select $B^{0} \rightarrow D^{-} \pi^{+}, \quad D^{-} \rightarrow K_{S}^{0} K^{-}$decays requiring $1.84<m\left(K_{S}^{0} K^{ \pm}\right)<1.89 \mathrm{GeV} / c^{2}$, and $B^{0} \rightarrow D^{-} K^{+}$, $D^{-} \rightarrow K_{S}^{0} \pi^{-} \quad$ decays requiring $1.84<m\left(K_{S}^{0} \pi^{ \pm}\right)<$ $1.89 \mathrm{GeV} / c^{2}$. We then apply our fit to find the yields for the $B^{0} \rightarrow D^{-} \pi^{+}$and $B^{0} \rightarrow D^{-} K^{+}$channels. We find values consistent with the expectations based on worldaverage product branching fractions [27] within statistical uncertainties.

Applying the fit method described above to the 14276 selected candidate $B^{0} \rightarrow K_{S}^{0} K^{ \pm} \pi^{\mp}$ events, we find $262 \pm$ 47 signal events. The fitted yields of the $B \bar{B}_{2}$ and $B \bar{B}_{3}$ categories are $199 \pm 51$ and $262 \pm 55$, respectively, consistent with the MC-based expectations. The fitted values of all other free parameters of the fit are also consistent with expectations based on studies of control samples and MC simulations. The results of the fit are shown in Fig. 1. The statistical significance of the signal yield, given by the square root of the difference between twice the value of negative log likelihood obtained assuming zero signal events to that at its minimum, is $6.0 \sigma$. Including systematic uncertainties (discussed below), the significance is $5.2 \sigma$.

The $B^{0} \rightarrow K_{S}^{0} K^{ \pm} \pi^{\mp}$ branching fraction is determined from the result of the fit by calculating signal probabilities for each candidate event with the ${ }_{s}$ Plot technique [33]. These are divided by event-by-event efficiencies that take the Dalitz-plot position dependence into account, and summed to obtain an efficiency-corrected signal yield of $1326 \pm 207$ events. We further correct for the effect of the charm and charmonium vetoes (estimated using a range of MC samples with different Dalitz-plot distributions), and divide by the total number of $B \bar{B}$ events in the data sample assuming equal production of $B^{0} \bar{B}^{0}$ and $B^{+} B^{-}$at the $\Upsilon(4 S)$. The result for the branching fraction is $\mathcal{B}\left(B^{0} \rightarrow\right.$ $\left.K_{S}^{0} K^{ \pm} \pi^{\mp}\right)=(3.2 \pm 0.5 \pm 0.3) \times 10^{-6}$, where the first error is statistical and the second is systematic.

We find the systematic error to be due to uncertainties in the signal PDFs (5.2\%), including possible data-MC differences in the signal PDF shapes evaluated using the control sample of $B^{0} \rightarrow D^{-} \pi^{+}, D^{-} \rightarrow K_{S}^{0} \pi^{-}$decays; uncertainties in the background PDFs $(2.5 \%)$, including effects due to the fixed values of the some of the $q \bar{q} \mathrm{PDF}$
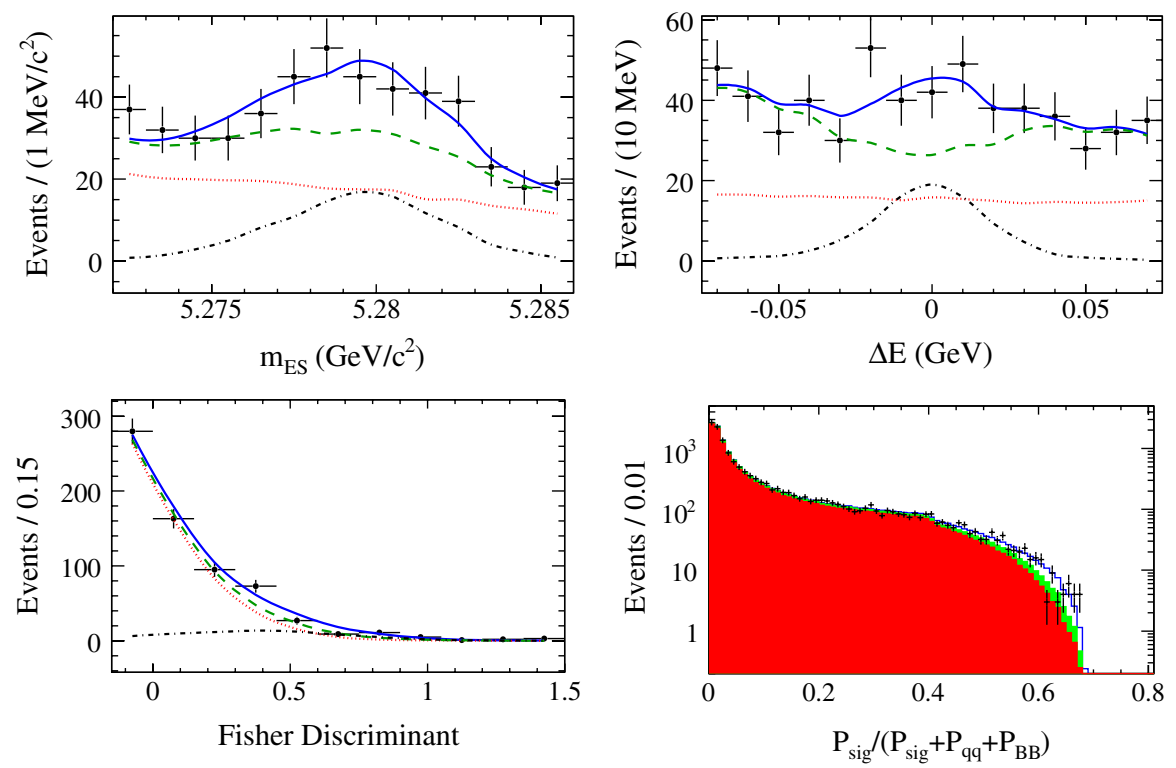

FIG. 1 (color online). Projections of candidate events onto (top left) $m_{\mathrm{ES}}$, (top right) $\Delta E$ and (bottom left) $\mathcal{F}$, following a requirement on the likelihood ratio to enhance signal visibility, calculated without the plotted variable. Points with error bars show the data, the solid (blue) curves are the total fit result, the dotted (red) curves are the $q \bar{q}$ component, and the dashed (green) curves show the total background contribution. The dash-dotted curves represent the signal contribution. Note that the $B \bar{B}_{2}$ and $B \bar{B}_{3}$ background categories have $\Delta E$ distributions that peak with negative and positive mean values, respectively. The bottom right plot shows the distribution of the likelihood ratio $\mathcal{P}_{\text {sig }} /\left(\mathcal{P}_{\text {sig }}+\mathcal{P}_{B \bar{B}}+\mathcal{P}_{q \bar{q}}\right)$ for all candidate events. Points with error bars show the data, the dark (red) filled area shows the contribution from $q \bar{q}$ background, the light (green) filled area shows the contribution from $B \bar{B}$ background, and the solid (blue) histogram shows the sum of all contributions. 
parameters (recall that the parametrization used is validated with off-peak and MC samples and that the most critical parameters are floated in the fit to data; the uncertainties are evaluated by varying the fixed parameters) and due to the fixed content of the histograms used to describe the $B \bar{B}$ background PDFs; potential fit biases, studied using ensembles of simulated experiments where continuum events are drawn from the PDF shapes and signal and $B \bar{B}$ background events are randomly extracted from MC samples $(1.1 \%)$; uncertainties in the efficiency due to tracking $(0.8 \%), K_{S}^{0}$ selection $(0.9 \%)$, and particle identification (2.8\%); and the error in the number of $B \bar{B}$ events $(1.1 \%)$. We assign two systematic uncertainties to account for the nonuniform Dalitz plot structure of the signal, both of which are estimated from MC simulations with different resonant contributions: uncertainty in the fraction of misreconstructed events (3.0\%) and uncertainty in the correction due to vetoes (4.1\%). Other sources of systematic uncertainty, including the fixed yields of $B \bar{B}_{1}$ and $B \bar{B}_{4}$, are found to be negligible (recall that the fitted yields of $B \bar{B}_{2}$ and $B \bar{B}_{3}$ are consistent with expectation).

In Fig. 2 we show the efficiency-corrected Dalitz plot for signal decays, obtained using event-by-event signal probabilities. We verify that this technique correctly reconstructs the signal Dalitz plot distribution using MC simulations in which the $B^{0} \rightarrow K_{S}^{0} K^{ \pm} \pi^{\mp}$ events contain different structures. There appears to be some structure in the $K^{* 0}$ region at low $K^{ \pm} \pi^{\mp}$ invariant mass, and an excess of events at low $K_{S}^{0} K^{ \pm}$mass with a highly asymmetric helicity angle distribution. Quantitative statements concerning the content of the Dalitz plot require a dedicated amplitude analysis, which is beyond the scope of the present study. However, it appears that there is no major contribution from an isospin partner of the $f_{\mathrm{X}}(1500)$ decaying to $K_{S}^{0} K^{+}$, which contrasts to the clear signal seen in $B^{+} \rightarrow K^{+} K^{-} \pi^{+}$decays [14].

In summary, using the full BABAR data sample of $424 \mathrm{fb}^{-1}$ collected at the $Y(4 S)$ resonance, we find evidence for charmless hadronic decays of neutral $B$ mesons to the final state $K_{S}^{0} K^{ \pm} \pi^{\mp}$. The signal has a significance of

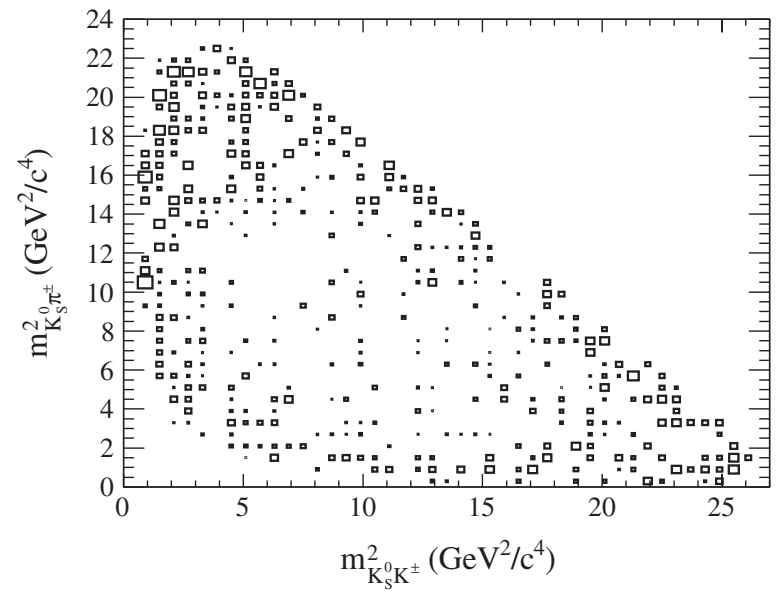

FIG. 2. Efficiency-corrected Dalitz plot distribution of $B^{0} \rightarrow$ $K_{S}^{0} K^{ \pm} \pi^{\mp}$ decays, obtained with the ${ }_{s}$ Plot technique [33]. Bins with negative content appear empty as do regions corresponding to the charm and charmonium vetoes. The area of each box is proportional to the number of weighted events in that bin; the largest bin corresponds to 12 events.

$5.2 \sigma$, after taking systematic effects into account. We measure the branching fraction to be $\mathcal{B}\left(B^{0} \rightarrow\right.$ $\left.K_{S}^{0} K^{ \pm} \pi^{\mp}\right)=(3.2 \pm 0.5 \pm 0.3) \times 10^{-6}$. We convert this result to $\mathcal{B}\left[B^{0} \rightarrow\left(K^{0} K^{+} \pi^{-}+\bar{K}^{0} K^{-} \pi^{+}\right)\right]=(6.4 \pm$ $1.0 \pm 0.6) \times 10^{-6}$ by multiplying by a factor of 2 .

We are grateful for the excellent luminosity and machine conditions provided by our PEP-II colleagues, and for the substantial dedicated effort from the computing organizations that support $B A B A R$. The collaborating institutions wish to thank SLAC for its support and kind hospitality. This work is supported by DOE and NSF (USA), NSERC (Canada), CEA and CNRS-IN2P3 (France), BMBF and DFG (Germany), INFN (Italy), FOM (The Netherlands), NFR (Norway), MES (Russia), MEC (Spain), and STFC (United Kingdom). Individuals have received support from the Marie Curie EIF (European Union) and the A. P. Sloan Foundation.
[1] B. Aubert et al. (BABAR Collaboration), Phys. Rev. Lett. 97, 171805 (2006).

[2] S. W. Lin et al. (Belle Collaboration), Phys. Rev. Lett. 98, 181804 (2007).

[3] B. Aubert et al. (BABAR Collaboration), Phys. Rev. Lett. 100, 081801 (2008).

[4] B. Aubert et al. (BABAR Collaboration), Phys. Rev. D 79, 051102 (2009).

[5] C. C. Chiang et al. (Belle Collaboration), Phys. Rev. D 81, 071101 (2010).
[6] B. Aubert et al. (BABAR Collaboration), Phys. Rev. D 74, 072008 (2006).

[7] B. Aubert et al. (BABAR Collaboration), Phys. Rev. D 76, 071103 (2007).

[8] B. Aubert et al. (BABAR Collaboration), Phys. Rev. D 75, 012008 (2007).

[9] T. Aaltonen et al. (CDF Collaboration), Phys. Rev. Lett. 103, 031801 (2009).

[10] B. Aubert et al. (BABAR Collaboration), Phys. Rev. D 78, 051103 (2008). 
P. DEL AMO SANCHEZ et al.

[11] B. Aubert et al. (BABAR Collaboration), Phys. Rev. D 76, 071104 (2007).

[12] B. Aubert et al. (BABAR Collaboration), Phys. Rev. D 78, 091102 (2008).

[13] B. Aubert et al. (BABAR Collaboration), Phys. Rev. D 74, 051104 (2006).

[14] B. Aubert et al. (BABAR Collaboration), Phys. Rev. Lett. 99, 221801 (2007).

[15] B. Aubert et al. (BABAR Collaboration), Phys. Rev. D 79, 051101 (2009).

[16] A. Garmash et al. (Belle Collaboration), Phys. Rev. D 69 , 012001 (2004).

[17] D. S. Du and L. B. Guo, Z. Phys. C 75, 9 (1997).

[18] A. Ali, G. Kramer, and C. D. Lu, Phys. Rev. D 58, 094009 (1998).

[19] Y.H. Chen et al., Phys. Rev. D 60, 094014 (1999).

[20] N. G. Deshpande, B. Dutta, and S. Oh, Phys. Lett. B 473, 141 (2000).

[21] D.S. Du et al., Phys. Rev. D 65, 094025 (2002); 66, 079904(E) (2002).

[22] M. Beneke and M. Neubert, Nucl. Phys. B675, 333 (2003).
PHYSICAL REVIEW D 82, 031101(R) (2010)

[23] C. W. Chiang et al., Phys. Rev. D 69, 034001 (2004).

[24] L. Guo, Q. G. Xu, and Z. J. Xiao, Phys. Rev. D 75, 014019 (2007).

[25] R. M. Wang et al., Eur. Phys. J. C 47, 815 (2006).

[26] B. Aubert et al. (BABAR Collaboration), Nucl. Instrum. Methods Phys. Res., Sect. A 479, 1 (2002).

[27] C. Amsler et al. (Particle Data Group), Phys. Lett. B 667, 1 (2008).

[28] B. Aubert et al. (BABAR Collaboration), Phys. Rev. D 79, 072006 (2009).

[29] B. Aubert et al. (BABAR Collaboration), Phys. Rev. Lett. 99, 161802 (2007).

[30] B. Aubert et al. (BABAR Collaboration), Phys. Rev. D 80, 112001 (2009).

[31] E. Barberio et al. (Heavy Flavor Averaging Group), arXiv:0808.1297, and online update at http://www.slac. stanford.edu/xorg/hfag/.

[32] H. Albrecht et al. (ARGUS Collaboration), Phys. Lett. B 241, 278 (1990).

[33] M. Pivk and F. R. Le Diberder, Nucl. Instrum. Methods Phys. Res., Sect. A 555, 356 (2005). 